Research Article

\title{
Isolation and Identification of Optochin-Resistant Viridans Group Streptococci from the Sputum Samples of Adult Patients in Jakarta, Indonesia
}

\author{
Wisiva Tofriska Paramaiswari $\left(\mathbb{D},{ }^{1}\right.$ Nurma Sumar Sidik $(\mathbb{D}),{ }^{2}$ Miftahuddin Majid Khoeri $(\mathbb{D})$, \\ Wisnu Tafroji $\left(\mathbb{1},{ }^{1}\right.$ Wahyu Finasari Said $\left(\mathbb{1},{ }^{3}\right.$ and Dodi Safari $\mathbb{C}^{1}$ \\ ${ }^{1}$ Eijkman Institute for Molecular Biology, Jakarta, Indonesia \\ ${ }^{2}$ Department of Biochemistry, Bogor Agricultural University, Bogor, Indonesia \\ ${ }^{3}$ Department of Clinical Pathology, Faculty of Medicine Universitas Indonesia/Cipto Mangunkusumo Hospital, \\ Jakarta, Indonesia
}

Correspondence should be addressed to Dodi Safari; safari@eijkman.go.id

Received 4 December 2020; Revised 20 February 2021; Accepted 5 July 2021; Published 14 July 2021

Academic Editor: Joseph Falkinham

Copyright (c) 2021 Wisiva Tofriska Paramaiswari et al. This is an open access article distributed under the Creative Commons Attribution License, which permits unrestricted use, distribution, and reproduction in any medium, provided the original work is properly cited.

\begin{abstract}
Aim. To investigate optochin-resistant viridans group streptococci (VGS) strains isolated from the sputum sample of adult patients with different clinical symptoms. Materials and Methods. Optochin-resistant VGS isolates were identified by matrixassisted laser desorption ionization time of flight mass spectrometry (MALDI-TOF MS). recA sequencing was used to confirm identified isolates at the genus level by MALDI-TOF MS. Finding. We identified 79\% of tested isolates (148/187) at the specieslevel identification using the MALDI-TOF MS tool. We identified that the most common species isolated from sputum specimens were S. oralis (44.9\%) followed by S. mitis (25.7\%), S. infantis (9.1\%), S. parasanguinis (7.5\%), S. peroris (3.7\%), S. anginosus (2.7\%), and S. sanguinis (2.1\%). Discussion. The S. oralis strains were majority of optochin-resistant VGS isolates obtained from sputum of adult patients in Jakarta, Indonesia. MALDI-TOF MS showed potential for the rapid identification tool to identify optochinresistant VGS isolates. Although there were discrepancies in identifying isolates at the genus/species level, the performance could be improved by expanding its database.
\end{abstract}

\section{Introduction}

The high-level similarities between Streptococcus pneumoniae, a human pathogen, and viridans group Streptococci (VGS), particularly within the nonpneumococcal mitis group including Streptococcus mitis, Streptococcus oralis, and Streptococcus pseudopneumoniae, often cause difficulties in species discrimination $[1,2]$. In clinical laboratory testing, conventional tests such as optochin sensitivity and bile solubility are still applied as key identifications for S. pneumoniae isolates [3]. However, some S. pneumoniae isolates were reported as optochin resistant in different geographical regions [4].
The VGS, a group of catalase-negative, Gram-positive Cocci, are a heterogeneous group of bacterium and considered to be normal flora of the oropharyngeal, urogenital, and gastrointestinal microbiota [5]. Classification of VGS has been challenging due to variability and overlap of their microbial characteristics [6]. This bacteria group includes a diverse range of organisms within the genus Streptococcus and can be characterized by green coloration on a blood agar plate [7]. Currently, VGS are classified into six major groups: the $S$. mutans group, S. salivarius group, S. anginosus group, $S$. mitis group, S. sanguinis group, and $S$. bovis group $[5,8]$. The pathogenicity of VGS ranges from opportunistic pathogens causing mild disease such as $S$. mutans that 
strongly correlates with dental caries development, and S. mitis, S. oralis, and S. sanguinis are taking roles in infective endocarditis [8].

Specific and accurate species-level identification of VGS is one of the important factors in patient clinical management and is also important for understanding their pathogenicity and virulence [1,9]. Matrix-assisted laser desorption ionization time of flight mass spectrometry (MALDI-TOF MS) has become an indispensable tool for clinical microbiology laboratories and shown to be a potential alternative for organism identification with a rapid and cost-saving method for VGS identification $[2,5,9]$. Previously, we reported that thirteen $S$. pneumoniae (pneumococcus) strains were susceptible to the optochin test and one hundred and eighty-nine of alpha haemolytic nonpneumococcus strains were resistant to the optochin test from the sputum of adult patients with nonspecific clinical symptoms in Jakarta, Indonesia [10]. In this study, we investigate further nonpneumococcus strains from adult patients for optochin-resistant VGS identification by the MALDI-TOF MS.

\section{Methods}

2.1. Streptococcus Group Collection. The Streptococcus group isolates were archived isolates obtained from sputum samples of adult patients with different clinical symptoms aged 18-87 years in Jakarta, Indonesia [10]. The patient clinical symptoms are tuberculosis $(n=51)$, community acquired pneumonia/healthcare-associated pneumonia $(n=17)$, SIDA/AIDS $(=10)$, diabetes mellitus $(n=6)$, pneumonia sepsis $(n=3)$, pneumonia $(n=2)$, other symptoms $(n=66)$, and missing data $(n=32)$. The sputum samples were inoculated onto blood agar plates supplemented with $5 \mathrm{mg} / \mathrm{L}$ of gentamicin and were incubated at $37^{\circ} \mathrm{C}$ in $5 \% \mathrm{CO}_{2}$ for $18-24 \mathrm{~h}$. All isolates that are alphahemolytic, resistant to optochin disk (ethylhydrocupreine hydrochloride), and insoluble in bile were included in this study [10].

2.2. Sample Preparation. All isolates were subcultured on a tryptone soya agar plate with $5 \%$ sheep blood and then incubated overnight at $37^{\circ} \mathrm{C}$ with $5 \% \mathrm{CO}_{2}$ [10]. A single colony of overnight pure growth bacteria was spotted to the MSP 96 ground plate (Bruker Daltonik, Germany) using a sterile toothpick and air dried in room temperature for approximately 5 minutes as a direct method sample preparation. The dried spots were then mixed with $1 \mu \mathrm{L}$ matrix (saturated solution of $\alpha$-cyano-4-hydroxycinnamic acid/ HCCA in $50 \%$ acetonitrile and $2.5 \%$ trifluoroacetic acid (TFA)). The solution was air dried in room temperature for approximately 10 mins. Standard protein extraction method was used to confirm the isolates with MALDI-TOF identification score $<2.000$ [11]. A $2.0 \mathrm{McF}$ arland of bacterial suspension was made in $300 \mu \mathrm{L}$ of water and then mixed with $900 \mu \mathrm{L}$ of ethanol. The suspension was homogenised and centrifuged at $20000 \times \mathrm{g}$ for 2 minutes. The supernatant was removed, and the pellet was dried at $55^{\circ} \mathrm{C}$ for
30 minutes. The dried pellet was resuspended in $50 \mu \mathrm{L}$ of acetonitrile followed by centrifugation at $20000 \times \mathrm{g}$ for 2 minutes. A $1 \mu \mathrm{L}$ supernatant was spotted to the ground plate and air dried for 10 mins in room temperature. Then, $1 \mu \mathrm{L}$ of matrix was added to the same spot as in the direct colony method as described above.

2.3. MALDI-TOF-MS-Based Identification. The isolates were identified using Microflex MALDI-TOF (Bruker Daltonik, Germany) and flexControl version 3.4 software as previously described $[2,12]$. Isolate identification was performed from spectrum acquisition was conducted in the positive linear mode with laser frequency at $60 \mathrm{~Hz}$. Mass range started at 2.000-20.000 Da. Each voltage from ion source 1 and ion source 2 was set at $20 \mathrm{kV}$ and $18.5 \mathrm{kV}$. Bacterial test standard protein was included in every test as instrument calibration. Automatic identification started after the spectra result was moved to Biotyper RTC software. The identification criteria were based on the similarity level, shown by the logarithmic score of isolates and database spectra prior to instructions by the manufacturer as follows: score $<1.700$ indicated isolates were not reliably identified; isolates with score 1.700-1.999 indicated identification accuracy up to the genus level; and isolates with score $\geq 2.000-3.000$ indicated isolates accurately identified up to the species level. Mass spectra analysis was conducted using flexAnalysis software. All obtained spectra were saved in flexControl before undergoing the calibration, smoothing, and baseline subtraction process on flexAnalysis MBT-Standard, prior to the manufacturer's recommendation.

2.4. recA Sequencing Identification. The recA sequencing tool was used to confirm all identified isolates at the genus level by MALDI-TOF MS [13]. We performed recA gene amplification and sequencing using forward primer [5'-GCCTTYATCGATGCBCARCA- $\left.3^{\prime}\right]$ and reverse primer [5'-GTTTC CGGRTTDCCRAACAT-3'] with the GoTaq Green Mastermix [13]. The obtained sequences were compared to the recA gene sequences database in NCBI GenBank and analysed using BLAST alignment (http://www.ncbi.nlm.nih.gov/blast) and MEGA-6 software. The obtained sequences with similarity $\geq 96 \%$ on published sequences in GenBank were assigned as cutoff for species identification.

\section{Results}

In this study, MALDI-TOF MS identified 79\% (148/187) isolates with score value ranging from $\geq 2.000-3.000$, indicating the highly probable species identification result. The majority identified species was $S$. oralis (50.7\%), followed by S. mitis (31.1\%), S. parasanguinis (9.5\%), S. anginosus (3.4\%), S. sanguinis (2.7\%), S. peroris (2.0\%), and S. pseudopneumoniae $(0.7 \%)$ (Table 1$)$. Meanwhile, we observed that $21 \%(39 / 187)$ of optochin-resistant VGS isolates were identified at the genus level (ID score value: 1.700-1.999) with majority isolates identified as S. oralis (38.5\%) followed by S. mitis $(23.1 \%)$, S. peroris (20.5\%), S. pneumoniae (12.8\%), S. parasanguinis (2.6\%), and S. infantis (2.6\%) (Table 2). The identification scores obtained from the isolates extracted using the standard 
TABLE 1: MALDI-TOF MS identification for viridans group Streptococcus strains isolates from sputum samples of adult patients.

\begin{tabular}{lcc}
\hline Species & \multicolumn{2}{c}{$\begin{array}{c}\text { MALDI-TOF MS identification } \\
\text { Probable genus }{ }^{\#}, n(\%)\end{array}$} \\
\hline S. infantis & Secure to highly probable species*, $n(\%)$ & $1(2.6)$ \\
S. anginosus & 0 & 0 \\
S. mitis & $5(3.4)$ & $9(23.1)$ \\
S. oralis & $46(31.1)$ & $15(38.5)$ \\
S. parasanguinis & $75(50.7)$ & $1(2.6)$ \\
S. peroris & $14(9.5)$ & $8(20.5)$ \\
S. pseudopneumoniae & $3(2.0)$ & 0 \\
S. sanguinis & $1(0.7)$ & 0 \\
S. pneumoniae & 4( & $5(12.8)$ \\
\hline
\end{tabular}

*Score values higher than $1.99 .{ }^{\#}$ Score values between 1.7 and 1.99 .

TABLE 2: Comparison of samples identified by MALDI-TOF with score 1.700-1.999 and recA sequence analysis.

\begin{tabular}{|c|c|c|}
\hline \multirow{2}{*}{ Isolate } & \multicolumn{2}{|c|}{ Identification methods } \\
\hline & MALDI-TOF MS (ID score value) & $r e c A$ sequencing (similarity score, \%) \\
\hline RIN 002 & S. infantis (1.942) & S. infantis $(96)$ \\
\hline RIN-176 & S. mitis (1.837) & S. infantis (93) \\
\hline RIN 289 & S. mitis (1.881) & S. infantis (99) \\
\hline RIN 106 & S. mitis (1.902) & S. infantis (96) \\
\hline RIN 230 & S. mitis (1.903) & S. mitis (98) \\
\hline RIN-267 & S. mitis (1.908) & S. mitis (94) \\
\hline RIN 241 & S. mitis (1.918) & S. oralis $(96)$ \\
\hline RIN-155 & S. mitis (1.931) & S. infantis (95) \\
\hline RIN-206 & S. mitis (1.940) & S. infantis/oralis (95) \\
\hline RIN 248 & S. mitis (1.993) & S. infantis (98) \\
\hline RIN-327 & S. oralis $(1.75)$ & S. infantis (93) \\
\hline RIN 312 & S. oralis $(1.797)$ & S. infantis (99) \\
\hline RIN 096 & S. oralis (1.833) & S. infantis (96) \\
\hline RIN-112 & S. oralis $(1.834)$ & S. infantis/oralis (95) \\
\hline RIN 215 & S. oralis $(1.860)$ & S. infantis (97) \\
\hline RIN 335 & S. oralis $(1.887)$ & S. infantis (95) \\
\hline RIN-221 & S. oralis $(1.898)$ & S. oralis $(94)$ \\
\hline RIN-114 & S. oralis (1.899) & S. oralis (94) \\
\hline RIN-089 & S. oralis (1.900) & S. oralis (95) \\
\hline RIN-132 & S. oralis (1.917) & S. peroris (94) \\
\hline RIN 129 & S. oralis (1.943) & S. oralis (99) \\
\hline RIN 083 & S. oralis (1.945) & S. oralis $(97)$ \\
\hline RIN 208 & S. oralis (1.947) & S. pneumoniae (97) \\
\hline RIN 025 & S. oralis $(1.951)$ & ND \\
\hline RIN 082 & S. oralis $(1.977)$ & S. oralis $(97)$ \\
\hline RIN-253 & S. parasanguinis (1.845) & Streptococcus sp. i-G2 (96) \\
\hline RIN-296 & S. peroris $(1.751)$ & S. peroris $(94)$ \\
\hline RIN-062 & S. peroris (1.775) & S. infantis (93) \\
\hline RIN-352 & S. peroris (1.877) & S. oralis $(93)$ \\
\hline RIN-113 & S. peroris (1.884) & S. infantis (95) \\
\hline RIN 052 & S. peroris (1.926) & S. infantis (99) \\
\hline RIN-188 & S. peroris (1.928) & S. infantis (94) \\
\hline RIN 190 & S. peroris (1.943) & S. infantis (97) \\
\hline RIN-142 & S. peroris (1.997) & S. peroris (95) \\
\hline RIN 022 & S. pneumoniae (1.834) & $\mathrm{ND}^{*}$ \\
\hline RIN 345 & S. pneumoniae (1.848) & S. oralis $(96)$ \\
\hline RIN 092 & S. pneumoniae (1.865) & S. infantis (97) \\
\hline RIN 320 & S. pneumoniae (1.896) & ND \\
\hline RIN-226 & S. pneumoniae (1.899) & S. peroris (95) \\
\hline
\end{tabular}

\footnotetext{
${ }^{*} \mathrm{ND}=$ not done.
} 
protein extraction method showed no significant difference with those using the direct colony method with the score ranging from 1.600-1.900 (data not shown).

We identified 11 optochin-resistant VGS isolates (30.6\%) at the genus level by the MALDI-TOF MS tool matched with the results from recA sequencing confirmation. S. oralis isolates were the most common matched isolates between MALDI-TOF MS and recA sequencing tools (six strains), followed by $S$. mitis and $S$. peroris (two strains each) and $S$. infantis (one strain). In this study, we observed that only one optochin-resistant VGS isolate was identified at the genus level by the MALDI-TOF MS tool (Table 1). After recA sequencing confirmation, 17 optochin-resistant VGS isolated at the genus level by the MALDI-TOF MS tool were identified as $S$. infantis (Table 2).

In total, we identified that the optochin-resistant VGS species isolated from sputum samples were S. oralis $(44.9 \%)$ followed by S. mitis (25.7\%), S. infantis (9.1\%), S. parasanguinis (7.5\%), S. peroris (3.7\%), S. anginosus (2.7\%), S. sanguinis $(2.1 \%)$, and others $(2.7 \%)$. S. oralis isolates were found to be higher in patients with age between 19 and 60 years compared to patients aged above 60 years (Figure 1). Meanwhile, S. infantis and S. parasanguinis were more often isolated from older patients than young patients. We also observed that $S$. oralis were more often isolated from sputum specimens of adult patients with community-acquired pneumonia/healthcare-associated pneumonia (64.7\%) and tuberculosis $(39.2 \%)$ symptoms (Figure 2).

\section{Discussion}

In this study, we found that $S$. oralis and $S$. mitis were the major common optochin-resistant VGS isolates (70.6\%) obtained from the sputum samples. The prevalence of S. mitis and S. oralis in this study was higher compared to other previous studies. Maeda et al. reported that the prevalence of $S$. mitis and $S$. oralis isolates from the sputum samples of adult patients with cystic fibrosis was $19 \%$ and $11 \%$, respectively [14]. Meanwhile, the S. anginosus group (38.8\%) and S. mitis (22.8\%) group were the most common VGS species isolated from bloodstream infection detected by MALDI-TOF MS identification [15]. From oncologic patients, almost half of the VGS isolated from the blood culture was S. mitis isolates (46.5\%) followed by S. anginosus (32.6\%) and S. sanguinis (16.3\%) by MALDI-TOF MS [6]. Oral streptococci isolates were reported as the most detected isolates from bronchoalveolar lavage fluid specimens obtained from pneumonia patients [16]. The oral streptococci isolates were all members of the $S$. mutans and $S$. mitis groups, the $S$. salivarius group, and the $S$. anginosus group except for S. pneumoniae [16]. S. mitis and S. oralis were significantly remaining species to be isolated from bloodstream isolates from neutropenic patients using the $\operatorname{sod} A$ gene detection [17].

In this study, we identified one isolate as S. infantis (2.6\%) at the genus level. However, more S. infantis (9.1\%) were identified from all optochin-resistant VGS isolates at the genus level by the MALDI-TOF MS tool after $\operatorname{rec} A$ sequencing confirmation. Zbinden A et al. reported that the A 313-bp part

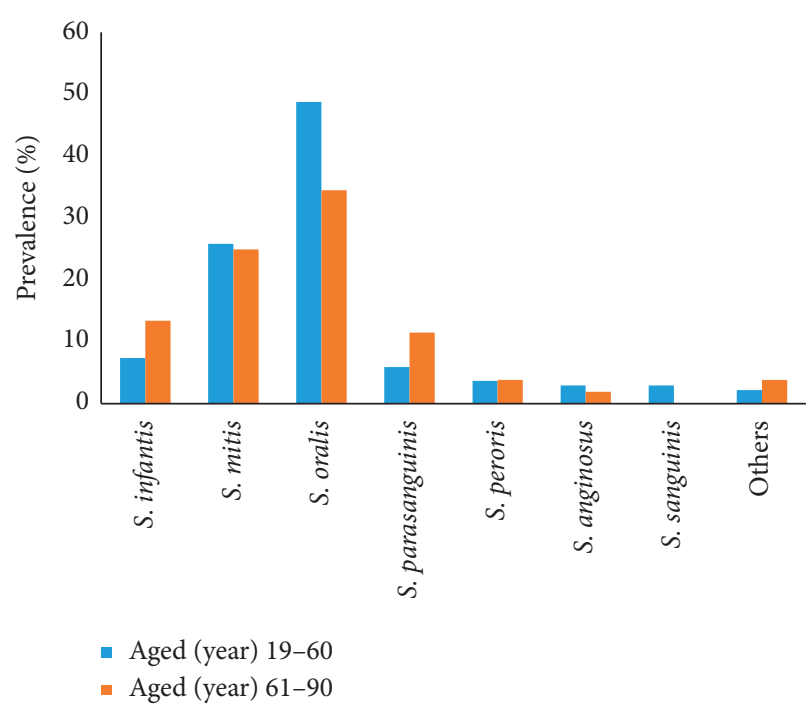

FIgURE 1: Prevalence of viridans group isolates isolated from adult patients with age 19 to 60 years (blue bar) and adult patients with age above 60 years (orange bar).

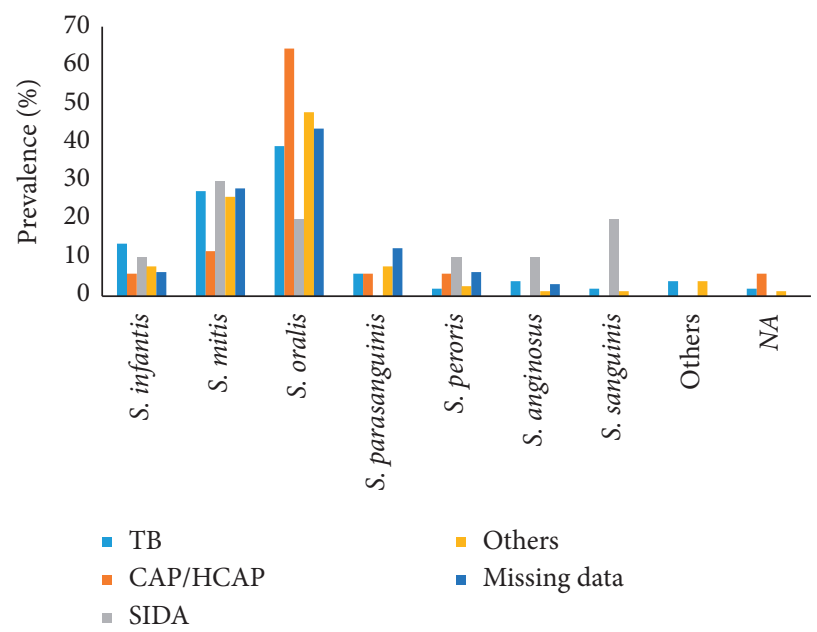

FIGURE 2: Prevalence of viridans group isolates isolated from adult patients with different clinical symptoms: tuberculosis (TB; blue bar), community-acquired pneumonia/healthcare-associated pneumonia (CAP/HCAP) (orange bar), SIDA/AIDS (gray bar), other symptoms (yellow bar), and missing data (blue dark bar).

of $r e c A$ was selected on the basis of variability within the $S$. mitis group, showing $<95.8 \%$ interspecies homology [13]. We found a mismatched pair of $S$. pneumoniae and $S$. infantis identified at the genus level by both MALDI-TOF MS and $r e c A$ sequencing tools. This discrepancy was possibly due to high similarities in the molecular and proteomic profile of the mitis group including $S$. mitis and $S$. oralis, thus presenting a challenge to correctly identify species using DNA- or protein-based identification methods $[2,18]$. The peak analysis and most updated Bruker database may improve the correct species identification [19]. In conclusion, the S. oralis and S. mitis were the predominant VGS isolates obtained from sputum of adult patients in Jakarta, Indonesia. MALDI-TOF MS showed potential for rapid identification to identify non-Streptococcus pneumoniae 
isolates. Although there were discrepancies in identifying isolates at the genus/species level, the performance could be improved by expanding its database.

\section{Data Availability}

The MALDI-TOF and recA sequencing data used to support the findings of this study are available from the corresponding author upon request.

\section{Disclosure}

The contents are solely the responsibility of the authors and do not necessarily represent the official views of the Centers for Disease Control and Prevention or the Department of Health and Human Services. The funders had no role in study design, data collection and analysis, decision to publish, or preparation of the manuscript.

\section{Conflicts of Interest}

The authors declare no conflicts of interest.

\section{Acknowledgments}

This research was partly funded by the Ministry of Research, Technology, and Higher Education, National Agency for Research and Innovation of the Republic of Indonesia, and was supported by the Cooperative Agreement, No. NU2GGH001852-03, funded by the Centers for Disease Control and Prevention, USA. The authors would like to thank Rahmadania Marita Joesoef for reviewing and English editing of this manuscript.

\section{References}

[1] L. N. Ikryannikova, A. V. Filimonova, M. V. Malakhova et al., "Discrimination between Streptococcus pneumoniae and Streptococcus mitis based on sorting of their MALDI mass spectra," Clinical Microbiology and Infection, vol. 19, no. 11, pp. 1066-1071, 2013.

[2] A. M. Werno, M. Christner, T. P. Anderson, and D. R. Murdoch, "Differentiation of Streptococcus pneumoniae from nonpneumococcal streptococci of the streptococcus mitis group by matrix-assisted laser desorption ionizationtime of flight mass spectrometry," Journal of Clinical Microbiology, vol. 50, no. 9, pp. 2863-2867, 2012.

[3] R. Y. Yahiaoui, C. D. Heijer, P. Wolfs, C. A. Bruggeman, and E. E. Stobberingh, "Evaluation of phenotypic and molecular methods for identification of Streptococcus pneumoniae," Future Microbiology, vol. 11, no. 1, pp. 43-50, 2016.

[4] M. Nagata, O. Ueda, T. Shobuike, T. Muratani, Y. Aoki, and H. Miyamoto, "Emergence of optochin resistance among Streptococcus pneumoniae in Japan," Open Journal of Medical Microbiology, vol. 2, no. 1, pp. 8-15, 2012.

[5] C. D. Doern and B. D. Carey-Ann, "It's not easy being green: the viridans group streptococci, with a focus on pediatric clinical manifestations," Journal of Clinical Microbiology, vol. 48, no. 11, pp. 3829-3835, 2010.

[6] F. Guerrero-Del-Cueto, C. Ibanes-Gutiérrez, C. VelázquezAcosta et al., "Microbiology and clinical characteristics of viridans group streptococci in patients with cancer," The
Brazilian Journal of Infectious Diseases, vol. 22, no. 4, pp. 323-327, 2018.

[7] Y. Maeda, C. E. Goldsmith, W. A. Coulter et al., "The viridans group streptococci," Reviews in Medical Microbiology, vol. 21, no. 4, pp. 69-79, 2010.

[8] C. Teles, A. Smith, G. Ramage, and S. Lang, "Identification of clinically relevant viridans group streptococci by phenotypic and genotypic analysis," European Journal of Clinical Microbiology \& Infectious Diseases, vol. 30, no. 2, pp. 243-250, 2011.

[9] S. Angeletti, G. Dicuonzo, A. Avola et al., "Viridans group streptococci clinical isolates: MALDI-TOF mass spectrometry versus gene sequence-based identification," Plos One, vol. 10, no. 3, Article ID e0120502, 2015.

[10] W. F. Said, E. Sukoto, M. M. Khoeri, J. Kumalawati, and D. Safari, "Serotype distribution and antimicrobial susceptibility of streptococcus pneumoniae isolates from adult patients in Jakarta, Indonesia," Journal of Infection and Public Health, vol. 10, no. 6, pp. 833-835, 2017.

[11] N. Matsuda, M. Matsuda, S. Notake et al., "Evaluation of a simple protein extraction method for species identification of clinically relevant staphylococci by matrix-assisted laser desorption ionization-time of flight mass spectrometry," Journal of Clinical Microbiology, vol. 50, no. 12, pp. 3862-3866, 2012.

[12] A. N. Suryatenggara, M. K. Miftahuddin, L. Waslia et al., "Identification and antibiotic susceptibility of methicillinresistant Staphylococcus aureus strains collected at a referral hospital, Jakarta, Indonesia in 2013," The Southeast Asian Journal of Tropical Medicine and Public Health, vol. 49, pp. 1053-1059, 2018.

[13] A. Zbinden, N. Köhler, and G. V. Bloemberg, "recA-based PCR assay for accurate differentiation of streptococcus pneumoniae from other viridans streptococci," Journal of Clinical Microbiology, vol. 49, no. 2, pp. 523-527, 2011.

[14] Y. Maeda, J. S. Elborn, M. D. Parkins et al., "Population structure and characterization of viridans group streptococci (VGS) including streptococcus pneumoniae isolated from adult patients with cystic fibrosis (CF)," Journal of Cystic Fibrosis, vol. 10, no. 2, pp. 133-139, 2011.

[15] T.-Y. Su, M.-H. Lee, C.-T. Huang, T.-P. Liu, and J.-J. Lu, "The clinical impact of patients with bloodstream infection with different groups of viridans group streptococci by using matrixassisted laser desorption ionization-time of flight mass spectrometry (MALDI-TOF MS)," Medicine, vol. 97, no. 50, Article ID e13607, 2018.

[16] K. Akata, K. Yatera, K. Yamasaki et al., "The significance of oral streptococci in patients with pneumonia with risk factors for aspiration: the bacterial floral analysis of $16 \mathrm{~S}$ ribosomal RNA gene using bronchoalveolar lavage fluid," BMC Pulmonary Medicine, vol. 16, no. 1, p. 16, 2016.

[17] T. Kitten, C. L. Munro, N. Q. Zollar, S. P. Lee, and R. D. Patel, "Oral streptococcal bacteremia in hospitalized patients: taxonomic identification and clinical characterization," Journal of Clinical Microbiology, vol. 50, no. 3, pp. 1039-1042, 2012.

[18] I. Harju, C. Lange, M. Kostrzewa, T. Maier, K. RantakokkoJalava, and M. Haanperä, "Improved differentiation of Streptococcus pneumoniae and other S. Mitis group streptococci by MALDI biotyper using an improved MALDI biotyper database content and a novel result interpretation algorithm," Journal of Clinical Microbiology, vol. 55, no. 3, pp. 914-922, 2017.

[19] M. Marín, E. Cercenado, C. Sánchez-Carrillo et al., "Accurate differentiation of Streptococcus pneumoniae from other species within the streptococcus mitis group by peak analysis using MALDI-TOF MS," Frontiers in Microbiology, vol. 8, p. 8, 2017. 